\title{
Neural mechanisms for prediction: do insects have forward models?
}

\section{Barbara Webb}

Institute for Perception, Action and Behaviour, School of Informatics, University of Edinburgh, James Clark Maxwell Building, King's Buildings, Mayfield Road, Edinburgh EH9 3JZ, UK

\begin{abstract}
'Forward models' are increasingly recognized as a crucial explanatory concept in vertebrate motor control. The essential idea is that an important function implemented by nervous systems is prediction of the sensory consequences of action. This is often associated with higher cognitive capabilities; however, many of the purposes forward models are thought to have analogues in insect behaviour, and the concept is closely connected to those of 'efference copy' and 'corollary discharge'. This article considers recent evidence from invertebrates that demonstrates the predictive modulation of sensory processes by motor output, and discusses to what extent this supports the conclusion that insect nervous systems also implement forward models. Several promising directions for further research are outlined.
\end{abstract}

A forward model is a mechanism that predicts the future state of a system given the current state and the control signals (Box 1). This concept is playing an increasing role in neuroscientific explanations of motor control, contextdependent action and cognition [1,2], as it is argued that biological systems need to be able to predict the sensory consequences of their actions in order to be capable of rapid, robust and adaptive behaviour. In vertebrate neuroscience, there is substantial interest in interpreting the function of various brain areas in these terms (e.g. the cerebellum $[3,4]$ ). Several authors have suggested that forward modelling could be a unifying framework for understanding the brain circuitry that underlies cognition [5-8].

Many of the problems that have motivated investigation of forward models in vertebrate neuroscience have close parallels in invertebrate neuroscience. For example, a cockroach executing a rapid escape response needs to know the current position of its legs in order to send the right motor command. It has been suggested that proprioceptive feedback might be too slow to serve this function, and that instead they maintain a prediction of their current position based on previous motor output [9] (although the sensory feedback loop in invertebrates is often much faster than in vertebrates and some cases extremely rapid, for example in the order of a millisecond [10]). Flying insects need to be able to distinguish selfinduced stimulation (such as rotation of the visual field

Corresponding author: Barbara Webb (bwebb@inf.ed.ac.uk). caused by tracking a target) from externally imposed stimulation (such as visual rotation caused by air disturbances) if they are to use the latter for flight stabilization [11]. This could be achieved by using motor outputs to predict expected visual input, and taking the difference from the actual input as a measure of the external disturbance. Locusts [12] and fruit flies [13] appear to adaptively adjust the gain parameters in their motor system to deal with alterations of the expected feedback when tested in flight simulators. These examples have often been addressed by reference to 'efference copy' or 'corollary discharge': terms that are closely related to forward models (Box 2).

The purpose of this article is to consider what sort of evidence might support the ascription of forward models to insects and other invertebrates, and to assess whether such evidence is available. If insects do have forward models they could provide convenient experimental systems for understanding the underlying neural mechanisms of prediction. However, if they do not, then we should be able to draw clear distinctions between aspects of vertebrate behaviour and neurophysiology that require such an interpretation, and those better explained by simpler alternatives.

\section{Is there central modulation of sensory processes?}

An important feature of forward modelling is that what the system is doing should modulate its sensory processing. That is, the perceptual system is not simply a feed-forward one, driven by the sensory input alone. There is ample evidence that the behavioural state of an insect can influence sensory and subsequent processing through neuromodulation. More interestingly, this can occur in a targeted fashion, i.e. specific sensory processes are enhanced or inhibited in a way that seems closely tied to the reafferent input that would be expected from the efferent output.

Poulet and Hedwig [14] have shown reduced responsiveness in auditory interneurons during singing in the male cricket (Figure 1). Gebhart and Honnegger [15] report, again in crickets, that interneurons sensitive to movement of the antennae are less sensitive during active movement by the cricket itself. It is well established that proprioceptive sensory neurons in several invertebrate systems, including crayfish [16], stick insects [17] and locusts [18,19], have responses that are modulated in phase with central pattern-generator rhythms. The 


\section{Box 1. What is a forward model?}

The term 'forward model' comes from control theory - the study of how to control the behaviour of dynamic systems. The principle is illustrated in Figure I. Consider a simple example, such as 'cruise control' in a car, where the aim is to control the speed of the vehicle with a motor command (normally given by the 'gas' pedal) that changes the throttle position. The change in wheel speed (the motor output) will be a complex function of factors such as the engine characteristics, the current speed and the coupling from engine to wheels. The change in speed of the car (the effect of the motor output on the world) will also depend on factors such as the road surface and the wind resistance. The actual speed can be measured by a sensor such as a tachometer, which itself has certain characteristics (the 'sensory input'). In theory, all these factors could be represented in a 'forward model' that takes the motor command as input and, by simulating the relevant processes, predicts the speed that will be measured by the tachometer.

Why not simply run the system and observe the consequences? There are several advantages to also making the prediction, as control engineers have discovered. One is the potential time lag involved in sensory feedback. For example, if the cruise controller waited until the tachometer reached the speed limit before closing the throttle, the adjustment could come too late to avoid exceeding the speed limit. Another is that a comparison between the prediction and the sensory input can be used to distinguish external disturbances from expected feedback. For example, if the car has a flat tyre, we would not want the controller to keep increasing output to try to reach the desired speed despite the additional friction, but rather to detect that some significant change to the system dynamics has occurred. Differences between prediction and feedback can also be used to learn and improve the generation of control signals that are, for example, well-tuned to the specific vehicle.

Another potential function of a predictor is that it can be run off-line to test out whether a specific control input is viable or leads to bad consequences (e.g. that changing the throttle position too rapidly might cause the engine to stall). This notion of a 'decoupled' forward model is an important one, but it should be noted that it is not an essential component of the forward model concept, at least as discussed in this paper. Claiming that a nervous system is using a forward model to predict is not, therefore, the same as claiming it is able to use it to plan.

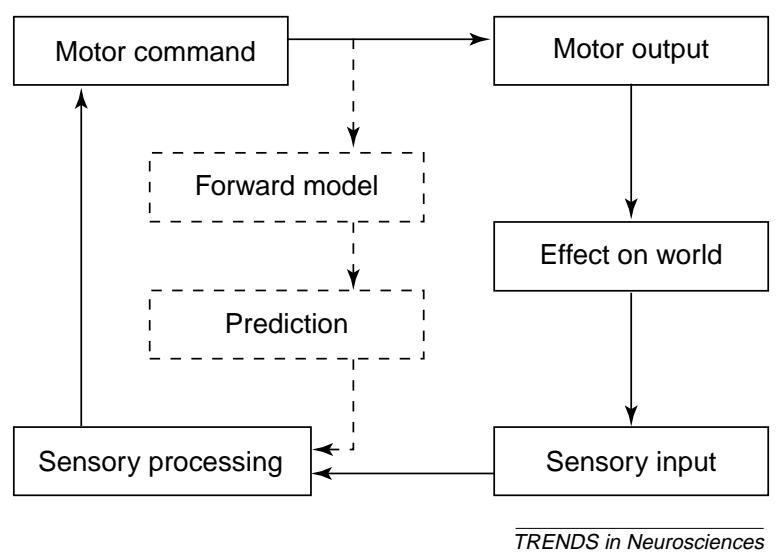

Figure I. Schematic representation of a sensory motor system with a forward model. The unbroken lines indicate the loop by which a motor command is translated into motor output, has some effect on the world and causes some sensory input, which the system can process to generate the next command. The forward model is an internal loop (broken lines) that takes the motor command and predicts the expected sensory input, which can be used to modulate the processing of the actual input. A classic example is that moving our eyes causes the image on the retina to move, but we perceive a stable world because the image movement is predictable from the eye movement command.

\section{Box 2. How do forward models relate to efference copy and corollary discharge?}

Descartes was perhaps the first to note explicitly that passive motion of the eye produces an impression of world motion when deliberate movements do not [39]. Pukyne and Helmholtz are usually credited as the first to suggest that this might involve motor output (or 'effort of will') being copied internally to interact with sensory input - that is, we do not see the world move if we can predict from the motor command to the eye that the scene is about to change - and Mach and von Uexkull were among the first to depict the idea in the form of feedback diagrams [39]. The concept was more clearly formulated in 1950, simultaneously and independently, as 'efference copy' by von Holst and Mittelstaedt [11] and as 'corollary discharge' by Sperry [40].

It is clear from reading the formulations of von Holst and Mittelstaedt and Sperry that they were describing the same principle as that outlined in Box 1 as forward modelling. Sperry describes corollary discharge 'into the visual centers [allowing] anticipatory adjustment...specific for each movement with regard to its direction and speed'. Von Holst and Mittelstaedt describe efference copy as being compared with the sensory re-afference in the same way as a 'photo-negative'. Sperry focuses on the advantages of being able to predict input in advance, whereas Von Holst and Mittlestaedt emphasize the potential for using the difference between the predicted and actual feedback to control behaviour. What is not so clear is whether 'efference copy' and 'corollary discharge' were intended to be the input to a forward model or the output of a forward model. If 'efference copy' means (as the name suggests) a copy of the efferent motor command, then this is the input to a forward model; but if 'efference copy' is to be compared as a 'photo-negative' to the sensory re-afference, then it must be the output of a forward model (i.e. the predicted sensory consequences of the motor command, not the motor command itself). 'Corollary discharge' is even more ambiguous: is it the discharge from the motor areas (the input to a forward model) or the discharge into the sensory areas (the output from a forward model)? This ambiguity reflects the tendency, still common in many discussions of efference copy and corollary discharge, to neglect the problem of how the motor output is to be transformed into a signal that can be compared with the sensory input. It is by no means obvious why these should be comparable. One advantage of the forward model terminology is that it makes this problem explicit.

Current research on forward models generally uses the term 'efference copy' to refer to the output of the motor command system that is fed into the predictor, and 'corollary discharge', where used at all, to refer to the output of the predictor (i.e. the signal fed into the sensory system). However, there is an alternative tradition in neuroethology that defines 'corollary discharge' as 'any neural signal that branches off centrally from an efference signal' [41]. 'Efference copy' is then taken to be that subset of corollary discharge in which the signal is 'exactly proportionate to the efference signal' and thus used to 'exactly counterbalance the reafference' [41] although, as already mentioned, it is not actually so evident that being proportionate to the motor signal will correctly predict the sensory signal. However, it is also not uncommon to find the terms 'efference copy' and 'corollary discharge' used interchangeably in invertebrate neuroethology.

alteration is not always suppression of the response; for example, Evans et al. [20] have reported on enhanced sensory neuron responses linked to motor rhythms in the Aplysia feeding network.

It could be argued that the specialized layout and peripheral processing characteristics of invertebrate sensors (characterized by Wehner [21] as 'neural models of the external world') are already a 'modulation' of the sensory system that optimizes it for the expected feedback from specific actions. An example is the receptive fields of horizontal cells in the fly visual system, which closely 


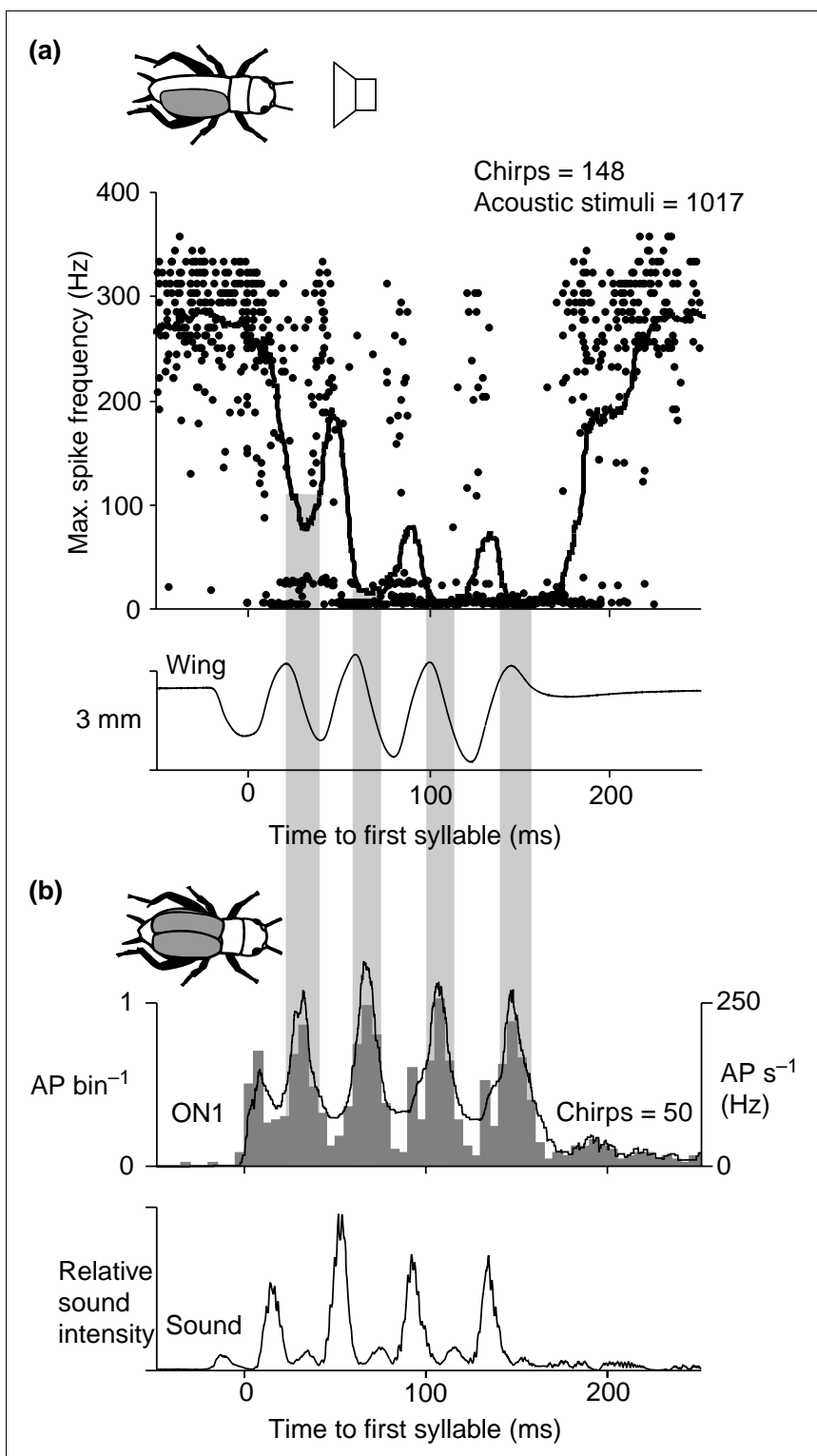

TRENDS in Neurosciences

Figure 1. Male crickets produce a calling song by rubbing one wing against the other, producing a series of sound bursts. The omega neuron (ON1) is an identified auditory interneuron in the prothoracic ganglion that normally has a strong response to the sound of cricket song, but this response is reduced for self-generated song. (a) If a male cricket has its own song made silent (by removing one wing) and is presented with an external acoustic stimulus, the spike frequency observed in ON1 to this stimulus is significantly reduced during silent singing movements (dots represent the maximum spike frequency; the black line represents the average spike frequency, plotted against the average wing movement). (b) During normal singing, the wing movements produce four bursts of sound (lower graph), which results in four action potential (AP) spike bursts in ON1 (shown as a peristimulus time histogram above) with a $15-20$ ms latency. The grey bars indicate that the response reduction seen in (a) is closely matched to the timing of the peaks of the response to self-generated sound seen in (b). Thus, the ON1 neuron of the male cricket will fire significantly less in response to its own song but will maintain sensitivity to external sources of sound. Reproduced, with permission, from Ref. [31].

resemble optical flow fields resulting from specific movements [22]. Alternatively, the animal might adopt a specific position or pattern of movement so as to enhance sensory data relevant to the specific task, such as the movements of the praying mantis that are used to extract depth information from parallax [23]. However, this type of tuned sensing and active perception, although interesting, can be distinguished from the forward-model concept by the following criteria.

\section{Is there evidence of internal connections from the motor system to the sensory areas?}

Motor output might affect sensory input via the environment or via proprioceptive feedback. But the implication of forward modelling is that there should be a specific output stream from motor areas that is routed not to muscles but to sensory areas. A useful example that illustrates the distinction is the modulation of wind-sensitive interneurons in the cockroach during flight [24]. Two pairs of identified 'giant' interneurons (GIs) in the terminal abdominal ganglion show different modulation. The ventral GIs have a strongly suppressed sensitivity, which appears in part to be caused by mechanical pinching of the nerve, because of the different position of the cerci during flight. The dorsal GIs show a strong rhythmic response linked to the wingbeat frequency, and this appears to have three different sources. One is proprioceptive feedback from the flight-linked oscillation of the cerci, one is external feedback caused by detection of the wind produced by each wingbeat and the last is a centrally generated motor signal, which is correlated with the motor output that passes from the thoracic ganglion down to the terminal abdominal ganglion [24]. Similarly, a combination of central and peripheral modulation of the GIs is found during walking [25].

Heterosynaptic modulation is also found in the much explored feeding mechanism in Aplysia. One sensory neuron in this circuit (B21) has been shown, for example, to have a variety of inhibitory and excitatory synaptic inputs, including interconnections from motoneurons that modulate its sensitivity [26]. However, it is, perhaps surprisingly, difficult to find many other examples where the specific connectivity from motor outputs to sensory inputs has been clearly confirmed. What is more common is the indirect evidence provided by demonstrating that the modulatory effect is still seen in the sensory systems when the motor output, and any potential proprioceptive feedback, has been prevented from occurring, for example, through de-afferentation. This was reported by Zaretsky and Rowell [27] as evidence of a corollary discharge for saccadic suppression in the locust. More recent studies have focused specifically on primary afferent depolarization (PAD) as an indicator of presynaptic inhibition of sensory inputs by motor outputs. For example, the biophysical mechanisms of $\mathrm{PADs}$ found in crayfish proprioception have been the subject of extensive study [28]. An interesting possibility is that the prediction is actually implemented by rather low-level properties, such as the precise synaptic positions of inhibitory inputs on the dendritic tree of the sensory neuron.

One question this discussion raises, however, is how to define 'motor output' and 'sensory area'. The simple boxand-arrow diagram (Figure I in Box 1), although conceptually convenient, is potentially misleading. In most biological systems, the generation of a motor command is a multistage process, and the 'efference copy' signal could branch off at any level, or indeed at several different levels. Similarly, sensory processing passes through several 
stages, and the predictive modulation might occur at any of these stages. Some areas of the nervous system combine sensory and motor functions. The forward-model 'loop' could be from the final motor neurons to the primary sensory afferents, or it could occur wholly within the brain of the animal. In fact it is possible to describe even a simple feedback mechanism as containing a predictor [29], as the goal state can be taken to be both a 'high-level' motor command and a prediction of the state that should result from executing the command: the difference of this from the actual feedback drives the behaviour. Yet this simple 'copying' of the goal does not seem to require any forwardmodel process to derive the prediction, which brings us to the next criterion.

\section{Does the modulation of sensory input by motor output involve non-trivial predictive processes?}

In the standard control theory paradigm, the forward model is constructed by representing the motor, environment and sensory mechanisms in sufficient detail to be able to predict the exact sensory consequences of a given motor command. In principle, an alternative to representing these processes explicitly would be to generate a lookup table in which each motor command is paired to an expected sensory input. In practice, an adequate prediction model or look-up table could be acquired by learning. Obviously, a complete and accurate model is rarely possible in practice (and seems unlikely to exist in the small brain of an insect), so any actual forward model is likely to be an approximation. However, there are many possible levels between accurate and detailed internal models, and simple approximate predictors. At what point on this continuum does the forward-model view (i.e. that the system implements a specific neural mechanism for calculating the predicted sensory input from the motor command) become unnecessary?

In many of the examples discussed so far, the modulation of the sensory input seems little more than the simple gating of a sensory channel, such as the inhibition of the optomotor response during escape turns, as seen in the locust [30]. This would not seem to require much calculation - although even this might require some prediction of the time-course of the expected input. There is likely, at the very least, to be a time lag between the motor output and the resulting sensory input, so any internal gating signal should be similarly delayed. The reafferent effects of a motor signal might often have a longer time course than the signal itself, in which case the gating needs to be similarly extended in duration. In some cases it can be shown that the timing and duration of inhibitory effects seen in insect sensory neurons are precisely matched to the predicted sensory feedback, for example, in cricket auditory perception (Figure 1). Male crickets produce bursts of sound by closing their wings. In auditory interneurons, an inhibitory current driven by efferent signals occurs with the same temporal pattern and with an appropriate delay, so as to reduce the response to each self-produced sound burst [31]. This appears to prevent auditory desensitization, so that the cricket remains sensitive to other males singing in the vicinity.
In the case of the male cricket, however, it appears that the amplitude of the inhibitory current is not scaled to the amplitude of the wing movements that produce the sound. It would be very interesting to discover whether more complex predictive transformations can be found in an invertebrate preparation. Another way to approach this issue is to look for behavioural evidence that an animal reacts to altered feedback in a manner consistent with a quantitative comparison between expected and actual feedback. This has been investigated to some extent using open- and closed-loop flight simulation in flies [13], and it has been suggested that they selectively ignore visual feedback in the expected direction but respond to feedback in the opposite direction. Employment of this paradigm is complicated by the possibility that the animal is able to adapt, during the experiment, to the altered feedback. More recent refinements in this experimental paradigm $[32,33]$ might enable more systematic investigation of these issues. For example, it would be interesting to attempt to implement analogues of some of the recent behavioural experiments on humans that have tested the forward-model hypothesis by using detailed temporal analysis of the behaviours [34,35].

Another interesting issue is whether it can be demonstrated in insects that the prediction is involved in learning or is modified by experience. Kanou et al. [36] show that crickets can adapt the directionality of their cercal escape response after damage only if they have experienced re-afferent input during free movement. The predictive mechanism required for more complex learnt behaviours might link various strands of evidence about the function of insect mushroom bodies. These distinctive structures in the insect brain are most often assumed to be principally involved in learning, particularly of olfactory discriminations [37]. But other lines of evidence suggest a role in multimodal integration and context-dependent behaviours. Evidence of efferent neurons that respond to specific stimuli combinations and sequences, and of recurrent connectivity, led $\mathrm{Li}$ and Strausfeld [38] to argue that the mushroom bodies might 'monitor motor actions or intended motor actions, differentiate self and imposed stimulation'. This is exactly the role suggested for forward models in the cerebellum of vertebrates.

\section{Concluding remarks}

There are several examples of recent research in invertebrate neuroscience that could benefit from, and have relevance to, the current discussions of forward modelling in vertebrate neuroscience. The link between specific physiological circuits and behaviour can potentially be more directly explored in these 'simpler' animals. Already the consideration of how we might determine whether and how prediction is used in these systems has been helpful to focus the often vague discussions of 'efferent copy' and 'corollary discharge' and to indicate what might be the most crucial lines of evidence to explore (Box 3). These can be summarized by asking whether central modulation of sensory processes in invertebrates can be demonstrated to be: (i) specifically targeted to enhance or suppress behaviourally relevant stimuli; (ii) well-scaled in amplitude and timing to match the expected feedback, 


\section{Box 3. Questions for future research}

- Can some of the behavioural experiments that have strongly supported the forward-model hypothesis for vertebrates be used as paradigms for invertebrate investigations?

- Can more examples of direct motor output to sensory areas be found, so that neural circuitry of these mechanisms can be understood?

- Are the mushroom bodies, or some other brain structure in insects, carrying out a non-trivial and learned prediction process?

- What are the implications for understanding cognitive processes in insects [42]?

demonstrating non-trivial transformation of the motor output to predict the input; and (iii) able to adapt to alterations in the motor-sensory linkages. Can we uncover the actual pathways and mechanisms by which the motor signal is transformed and transported so as to interact with the sensory input?

\section{References}

1 Wolpert, D.M. and Ghahramani, Z. (2000) Computational principles of movement neuroscience. Nat. Neurosci. 3, 1212-1217

2 Kawato, M. (1999) Internal models for motor control and trajectory planning. Curr. Opin. Neurobiol. 9, 718-727

3 Miall, R.C. et al. (1996) Sensory prediction as a role for the cerebellum. Behav. Brain Sci. 19, 466-467

4 Bell, C. (1995) The generation of expectations in cerebellum-like structures. In The Neurobiology of Computation: Proceedings of the Annual Computational Neuroscience Meeting (Bower, J., ed.), Klauer Associates

5 Clark, A. and Grush, R. (1999) Towards a cognitive robotics. Adapt. Behav. 7, 5-16

6 Barsalou, L. (1999) Perceptual symbol systems. Behav. Brain Sci. 22, 577-609

7 Grush, R. The emulation theory of representation: motor control, imagery, and perception. Behav. Brain Sci. (in press).

8 Crüse, H. (2003) The evolution of cognition - a hypothesis. Cognit. Sci. 27, 135-155

9 Camhi, J.M. and Levy, A. (1988) Organization of a complex movement: fixed and variable components of the cockroach escape behaviour. J. Comp. Physiol. A Sens. Neural. Behav. Physiol. 163, 317-328

10 Höltje, M. and Hustert, R. (2003) Rapid mechano-sensory pathways code leg impact and elicit very rapid reflexes in cockroaches. J. Exp. Biol. 206, 2715-2724

11 von Holst, E. and Mittlestaedt, H. (1950) Das Reafferenzprinzip. Naturwissenschaften 37, 464-476

12 Möhl, B. (1989) 'Biological noise' and plasticity of sensorimotor pathways in the locust flight system. J. Comp. Physiol. A Sens. Neural. Behav. Physiol. 166, 75-82

13 Heisenberg, M. and Wolf, R. (1988) Reafferent control of optomotor yaw torque in Drosophila melanogaster. J. Comp. Physiol. A Sens. Neural. Behav. Physiol. 163, 373-388

14 Poulet, J.F. and Hedwig, B. (2002) A corollary discharge maintains auditory sensitivity during sound production. Nature 418, 872-876

15 Gebhart, M. and Honnegger, H-W. (2001) Physiological characterisation of antennal mechanosensory descending interneurons in an insect (Gryllus bimaculatus, Gryllus campestris) brain. J. Exp. Biol. 204, 2265-2275

16 El Manira, A. et al. (1991) Monosynaptic interjoint reflexes and their central modulation during fictive locomotion in crayfish. Eur. J. Neurosci. 3, 1219-1231

17 Bassler, U. and Buschges, A. (1998) Pattern generation for stick insect walking movements - multisensory control of a locomotor program. Brain Res. Rev. 27, 65-88

18 Buschges, A. and Wolf, H. (1999) Phase dependent presynaptic modulation of mechanosensory signals in the locust flight system. J. Neurophysiol. 81, 959-962

19 Burrows, M. (1996) The Neurobiology of an Insect Brain, Oxford University Press

20 Evans, C.G. et al. (2003) Regulation of spike initiation and propagation in an aplysia sensory neuron: gating-in via central depolarization. J. Neurosci. 23, 2920-2931

21 Wehner, R. (1987) Matched filters: neural models of the external world. J. Comp. Physiol. A Sens. Neural. Behav. Physiol. 161, 511-531

22 Egelhaaf, M. et al. (2002) Neural encoding of behaviourally relevant visual-motion information in the fly. Trends Neurosci. 25, 96-102

23 Poteser, M. and Kral, K. (1995) Visual distance discrimination between stationary targets in praying mantis: an index of the use of motion parallax. J. Exp. Biol. 198, 2127-2137

24 Liberstat, F. et al. (1989) Multiple feedback loops in the flying cockroach: excitation of the dorsal and inhibition of the ventral giant interneurons. J. Comp. Physiol. A Sens. Neural. Behav. Physiol. 165, 651-668

25 Daley, D.L. and Delcomyn, F. (1980) Modulation of the excitability of cockroach giant interneurons during walking. II. Central and peripheral components. J. Comp. Physiol. A Sens. Neural. Behav. Physiol. 138, 241-251

26 Rosen, S.C. et al. (2000) Outputs of radula mechanoafferent neurons in Aplysia are modulated by motor neurons, interneurons, and sensory neurons. J. Neurophysiol. 83, 1621-1636

27 Zaretsky, M. and Rowell, C.H.F. (1979) Saccadic suppression by corollary discharge in the locust. Nature 280, 583-585

28 Cattaert, D. et al. (2001) Presynaptic inhibition and antidromic spikes in primary afferents of the crayfish: a computational and experimental analysis. J. Neurosci. 21, 1007-1021

29 Karniel, A. (2002) Three creatures named 'forward model'. Neural Netw. 15, 305-307

30 Robert, D. and Rowell, C.H.F. (1992) Locust flight steering 2: Acoustic avoidance maneuvers and associated head movements, compared with correctional steering. J. Comp. Physiol. A Sens. Neural. Behav. Physiol. 171, 53-62

31 Poulet, J.F.A. and Hedwig, B. (2003) A corollary discharge mechanism modulates central auditory processing in singing crickets. J. Neurophysiol. 89, 1528-1540

32 Kern, R. et al. (2001) Neuronal processing of behaviourally generated optic flow: experiments and model simulations. Network 12, 352-369

33 Sherman, A. and Dickinson, M.H. (2003) A comparison of visual and haltere-mediated equilibrium reflexes in the fruit fly Drosophila melanogaster. J. Exp. Biol. 206, 295-302

34 Wolpert, D.M. et al. (1995) An internal model for sensorimotor integration. Science 269, 1880-1882

35 Mehta, B. and Schaal, S. (2002) Forward models in visual control. J. Neurophysiol. 88, 942-953

36 Kanou, M. et al. (2002) Rearing conditions required for behavioral compensation after unilateral cercal ablation in the cricket Gryllus bimaculatus. Zool. Sci. 19, 403-409

37 Heisenberg, M. (1998) What do mushroom bodies do for the insect brain? Learn. Mem. 5, 1-10

38 Li, Y.S. and Strausfeld, N.J. (1999) Multimodal efferent and recurrent neurons in the medial lobes of cockroach mushroom bodies. J. Comp. Neurol. 409, 647-663

39 Grüsser, O-J. (1995) On the history of the ideas of efference copy and reafference. Clio Med. 33, 35-55

40 Sperry, R.W. (1950) Neural basis of the spontaneous optokinetic response produced by visual inversion. J. Comp. Physiol. Psychol. 43, 482-489

41 Camhi, J.M. (1984) Neuroethology: Nerve Cells and the Natural Behaviour of Animals, Sinauer Associates

42 Menzel, R. (1999) Cognition by a minibrain. Science 400, 718-719 